Franco Mongini

Secondo Fassino

Eugenia Rota

Andrea Deregibus

Maura Levi

Domenico Monticone

Giovanni Abbate-Daga

\section{The Temperament and Character Inventory in women with migraine}

Published online: 20 July 2005

F. Mongini $(\varangle) \cdot$ E. Rota $\bullet$ A. Deregibus

D. Monticone

Department of Clinical Pathophysiology,

Headache and Facial Pain Unit,

University of Turin, Corso Dogliotti 14,

I-10126 Turin, Italy

e-mail: franco.mongini@unito.it

Tel.: +39-011-6334041

Fax: +39-011-6636489

S. Fassino • M. Levi • G. Abbate-Daga

Department of Neuroscience,

Psychiatric Institute,

University of Turin, Turin, Italy

\begin{abstract}
The purpose of this work was to investigate: (1) the differences in temperament and character between 49 women with migraine and 49 controls using the Temperament and Character Inventory (TCI), and (2) the extent to which these differences were related to migraine or to the presence of comorbid depression. The migraine patients scored significantly higher than the controls in two temperament dimensions - Harm Avoidance (HA) and Persistence $(\mathrm{P})$ - and significantly lower in one character dimension - Self-Directedness (SDir) (Student's t). After multiple logistic regression, the TCI P and HA dimensions were
\end{abstract}

significantly associated with the presence of migraine. The HA dimension was also related to the presence of depression. Our results show that in migraine the higher HA score could be partly associated to comorbid depression while the high $\mathrm{P}$ dimension seems to be solely related to the presence of migraine.

Key words Headache • TCI • Migraine - Depression • Obsessiveness

\section{Introduction}

The Temperament and Character Inventory (TCI) [1] is by far the most used instrument to assess the temperament and character features of personality. It consists of seven independent dimensions. Four of these test temperament: Novelty Seeking (NS), Harm Avoidance (HA), Reward Dependence (RD) and Persistence (P). Briefly, NS expresses the level of activation of exploratory activity, HA reflects the efficiency of the behavioural inhibition system, RD is related to the maintenance of rewarded behaviour, while $\mathrm{P}$ expresses maintenance of behaviour as resistance to frustration. These temperamental traits appear to be mediated by different neurobiological mechanisms: NS by the dopaminergic system, HA by the serotoninergic system, RD by the noradrenergic system and $\mathrm{P}$ by the glutamatergic system [1]. The other three dimensions of the TCI are considered as personality traits acquired through experience: Self-Directedness (SDir), Cooperativeness (C) and Self-Transcendence (ST). SDir expresses the degree to which the self is viewed as autonomous and integrated, $\mathrm{C}$ reflects the extent to which the self is viewed as a part of society and ST expresses the degree to which the self is viewed as an integral part of the universe.

Although the TCI may be regarded as a promising instrument to assess personality in different headache types and their relation to the pathophysiological background of the disease, relatively little and conflicting evidence has been available until now. Nylander et al. [2] found in migraine 
patients no significant differences in higher order dimensions of temperament or character in comparison with the controls. However, all patients in this study belonged to the same Swedish migraine family with an autosomal dominant inheritance pattern and were, as such, rather selected. Di Piero et al. [3] compared patients with migraine and tension-type headache (TTH) with normative data and found high HA levels in both groups and high $\mathrm{P}$ and low NS levels in migraine patients only. Recently, Boz et al. [4] compared the data from the TCI and the Beck Depression Inventory (BDI) in patients with TTH and migraine and found that BDI scores were positively correlated with HA scores and negatively with SDir; moreover, multivariate analysis using the BDI score as a covariate showed significantly higher HA scores than the controls in TTH patients but not in migraine patients.

TCI dimensions have also been proven to be altered in mood disorders. As mood and anxiety disorders are a frequent finding in headache patients [5-8], the question arises as the extent to which the differences in TCI dimensions observed in migraine patients depend on the headache pathology or on the presence of depression. Both pathologies are indeed characterised by the involvement of the serotoninergic system.

The purpose of this work was to apply the TCI to a group of women with migraine in order to:

1. investigate the differences between migraine patients and normal controls, similar for age and education, in TCI temperament and character dimensions; and

2. evaluate the extent to which these differences were related to migraine or to the presence of depression.

\section{Subjects and methods}

Forty-nine females, aged 18-65 years, with episodic migraine were enrolled. The control group consisted of 47 healthy women with similar characteristics of age and education, but not suffering either from headache, or from other relevant medical or psychiatric disorders.
Every patient underwent a psychological assessment on the Axis I of the DSM-IV by means of a structured interview (SCID-1) [9]. The TCI was administered in the Italian validated version [10] to the migraine sufferers, in a headache-free period, and to the controls.

Significant score differences between the patient group and the controls were assessed (Student's $t$-test). To examine the extent to which the TCI scales could relate to the presence of migraine, odds ratio and confidence intervals (CI) at $95 \%$ were calculated in a multiple logistic regression model, taking the presence of migraine as a dependent variable and the TCI scores and age as independent variables. Then, to examine the relationship between TCI scales and depression, the same was done for the migraine patients, taking the presence of depression as a dependent variable. To examine the extent to which headache frequency and history could affect the TCI dimensions, a linear regression model was employed, taking the headache frequency and the years of headache as independent variables and the TCI scores as dependent variables.

\section{Results}

The migraine group scored significantly higher than the controls in HA and P, and significantly lower in SDir; no difference was found between patients and controls regarding the other temperament and character dimensions (Table 1).

There were $29(59.2 \%)$ patients with major depression as a comorbid disorder. In 25 of them an anxiety disorder was also present. The absence of other specific psychiatric disorders in our files may be attributed to the fact that patients with these disorders are more frequently referred directly to a psychiatric institution.

After multiple logistic regression, taking the presence of migraine as a dependent variable and the TCI scores and age as independent variables, statistically significant values were detected for the HA $(p=0.021$, odds ratio=1.13) and $\mathrm{P}$ $(p<0.001$, odds ratio $=1.76)$ dimensions. In migraine patients, after taking the presence of depression as a dependent variable and the TCI scores as independent variables, significant $p(0.14)$ and odds ratio (1.25) values were found for HA but

Table 1 TCI scores (mean \pm standard deviation) in migraine patients and controls

\begin{tabular}{lllc}
\hline & $\begin{array}{c}\text { Patients, } \\
\text { mean } \pm \text { SD }\end{array}$ & $\begin{array}{c}\text { Controls, } \\
\text { mean } \pm \text { SD }\end{array}$ & $p$ \\
NS & $16.61 \pm 5.27$ & $18.55 \pm 5.53$ & 0.175 \\
HA & $21.98 \pm 6.94$ & $16.79 \pm 6.69$ & $<0.001$ \\
RD & $15.14 \pm 3.45$ & $15.55 \pm 2.81$ & 0.233 \\
P & $5.20 \pm 1.86$ & $4.04 \pm 1.69$ & $<0.05$ \\
SDir & $25.71 \pm 6.76$ & $29.72 \pm 6.90$ & $<0.05$ \\
C & $30.55 \pm 5.16$ & $31.87 \pm 3.95$ & 0.851 \\
ST & $15.12 \pm 7.10$ & $15.64 \pm 6.56$ & 0.775 \\
\hline
\end{tabular}

$N S$, Novelty Seeking; HA, Harm Avoidance; $R D$, Reward Dependence; $P$, Persistence; SDir, Self-Directedness; $C$, Cooperativeness; $S T$, Self-Transcendence 
not for P. After linear regression, no significant relationship was found between the headache frequency and the years of headache and HA dimension ( $p=0.95, p=0.95$ respectively) and $\mathrm{P}$ dimension ( $p=0.20, p=0.62$ respectively) of the TCI.

\section{Discussion}

We found significant differences between patients and controls in three dimensions, two pertaining to the temperament (HA and P) and the third pertaining to the character (SDir). In particular, migraine patients showed significantly higher scores of HA and P and significantly lower scores in SDir.

Highly HA individuals are described as extremely careful, passive, fearful, insecure and prone to react with a high rate of anxiety and depression to stressful events, while high $P$ levels express the tendency to maintain unrewarded behaviours and correlate with rigidity and obsessiveness [1]. Low levels of SDir are an important predictor of categorical diagnosis of DSM Axis I disorders which are frequently associated with migraine [5-8].

The results of this study are consistent with those of a recent work [11], in which we administered the Object Alternation Test (OAT) to assess the function of the orbitofrontal cortex [12], in a group of women with chronic migraine. In the OAT the patient is asked to find a coin located under one of two identical boxes. His task is to learn that the box under which the coin is located is alternated after each correct response. Any trial with more than two consecutive wrong choices is considered as a "perseverative error". In our patients we found a perseverative error score four times higher than the controls. This finding is similar to what Cavedini et al. [13] observed in patients with obsessive compulsive disorder. The high $\mathrm{P}$ we found in our study and its relationship to the presence of migraine seems to confirm a specific tendency of these patients to develop some levels of rigidity and obsessiveness.

Our findings agree with those of Di Piero et al. [3], who observed elevated HA scores in patients with both migraine and $\mathrm{TTH}$, and significantly higher $\mathrm{P}$ in migraine patients only. Moreover, the results of the multiple logistic regression model suggest a direct relationship between the presence of migraine and the HA and P dimension of the TCI. While in migraine patients a higher HA dimension may also be partly related to the presence of depression as a comorbid disorder, in the case of the $\mathrm{P}$ dimension such association seems to be independent from this factor, as the multiple logistic regression model did not show a relationship when depression was taken as a dependent variable.

In conclusion, our results show that the TCI is a valuable instrument to reveal changes in temperament and character with a more biological adherence as compared to other commonly used questionnaires.

\section{References}

1. Cloninger CR, Przybeck TR, Svrakic DM, Wetzel RD (1994) The Temperament and Character Inventory (TCI): a guide to its development and use. Center for Psychobiology of Personality, Washington University, St. Louis, MO

2. Nylander PO, Schelette P, Brandstrom $S$ et al (1996) Migraine: temperament and character. J Psychiatr Res 30[Suppl 5]:359-368

3. Di Piero V, Bruti G, Venturi P et al (2001) Aminergic tone correlates of migraine and tension-type headache: a study using the tridimensional personality questionnaire. Headache 41 [Suppl 1]:63-71

4. Boz C, Velioglu S, Ozmenoglu M, Sayar K, Alioglu Z, Yalman B, Tobpas M (2004) Temperament and character profiles of patients with tension-type headache and migraine. Psychiatry Clin Neurosci 58:536-543
5. Merikangas KR (1994)

Psychopathology and headache syndromes in the community. Headache 34:S17-22

6. Breslau N, Davis GC, Schultz LR, Peterson EL (1994) Migraine and major depression: a longitudinal study. Headache 34:387-393

7. Mongini F, Keller R, Deregibus A, Raviola F, Mongini T, Sancarlo M (2003) Personality traits, depression and migraine in women. A longitudinal study. Cephalalgia 23:186-192

8. Franchini L, Bongiorno F, Dotoli D, Rainero I, Ponessi L, Smeraldi E (2004) Migraine headache and mood disorders: a descriptive study in an outpatient psychiatric population. J Affect Disord 81:157-160

9. Mazzi F, Morosini P, De Girolamo G, Lussetti M, De Guaraldi GP (2000) SCID-I. Structured Clinical Interview for DSM-IV Axis I Disorders (Italian Version). OS-Organizzazioni Speciali, Firenze
10. Manfredonia MG, Gasperini M, Sciuto G, Bellodi L, Smeraldi E (1991) The distribution of dimensional personality traits in an Italian general population. New Trends Exp Clin Psychiatr 7:113-129

11. Mongini F, Keller R, Deregibus A, Barbalonga E, Mongini T (2005) Frontal lobe dysfunction in patients with chronic migraine. Psychiatry Res 113:101-106

12. Mishkin M, Vest B, Waxler M, Rosvold E (1969) A re-examination of the effects of frontal lesions on objects alternation. Neuropsychologia 7:357-363

13. Cavedini P, Ferri S, Scarone S, Bellodi L (1998) Frontal lobe dysfunction in obsessive-compulsive disorder and major depression: a clinical-neuropsychological study. Psychiatry Res 78:21-28 